\section{PS039 希土類材料を用いた膜蛋白質の高感度イメージング}

Highly sensitive imaging of cell membrane proteins by using lanthanide materials

Tatsuya Nakamrua ${ }^{1}$, Shin Mizukami ${ }^{1.2}$, Kazuya Kikuchi ${ }^{1,2}$ ('Graduate school of Engineering, Osaka University, ${ }^{2}$ Immunology Frontier Research Center, Osaka University)

Since cell membrane proteins play important roles for membrane transport and signaling, it is important to analyze functions of cell membrane proteins. So far, fluorescent proteins have been utilized to study the protein behaviors. On the other hand, protein labeling technique with organic dyes and protein tags has been focused because it yields temporal labeling and excellent fluorescent properties. Our group developed protein labeling system based on a mutant $\beta$ lactamase (BL-tag), which specifically forms covalent binding with $\beta$-lactam compounds. We have developed the fluorogenic probes, which consist of organic fluorescent dyes and $\beta$-lactam compound.

In this study, we further developed new probes that utilize luminescent lanthanide complex with silica nanoparticles to achieve higher measurement sensitivity. Luminescent lanthanide complexes show long lifetime luminescence that reaches millisecond order. Since fluorescence lifetime of organic dyes is of the order of nanoseconds, luminescent lanthanide complexes enable timeresolved fluorescence measurement to eliminate short-lifetime fluorescence such as autofluorescence from biological samples. Silica nanoparticles are biocompatible materials and can include a large number of dyes. By combining lanthanide complex with silica nanoparticles, we developed highly luminescent lanthanide silica nanoparticles. Additionally, we attempted labeling of BL-tag expressed on cell surface.

\section{PS040 ストレス顆粒内 mRNA 微小樌造体の超解像イメージング}

Super-resolution imaging of mRNA nano-structures in stress granule

Ko Sugawara, Kohki Okabe, Akihiko Sakamoto, Takashi Funatsu (Grad. Sch. Pha. Sci., Univ. Tokyo)

mRNAs play critical roles in gene expression with various regulations. Under stress, cytoplasmic mRNAs assemble and form stress granules (SGs), where they are remodeled for repression of translation. However, the mechanisms of action remain elusive. The aim of this study was to visualize the fine structure of SGs and to investigate the detailed physiological role of them. Stochastic optical reconstruction microscopy (STORM) provided us super-resolution images with spatial resolution of $\sim 20 \mathrm{~nm}$ in the lateral direction and of $\sim 60 \mathrm{~nm}$ in the axial direction. Three-dimensional super-resolution imaging was performed using cylindrical lens. To visualize endogenous cytoplasmic mRNAs, we have microinjected Cy5-labeled linear antisense $2^{\prime}-O$-methyl probes into the cytoplasm of $\operatorname{COS} 7$ cells. After the injection, cellular stress was induced by addition of $0.5 \mathrm{mM}$ arsenite in a culture medium. To examine the maturation of SGs, STORM images were captured at various time-points during SG formation. Three-dimensional super-resolution images showed that endogenous mRNAs located in spherical compartments with a diameter of $200 \mathrm{~nm}$, which could not be observed in diffraction-limited imaging. We termed this structure "mini-granule". With stress duration, mini-granules increased in number, while they maintained the same size. The results demonstrated that the growing process of SGs was resulted from the assembly of mini-granules. The result of this study indicated that mini-granules were responsible for the physiological functions of SGs.

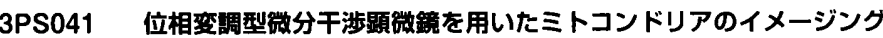 解析}

The imaging analysis of mitochondria with retardation mediated differential interference microscope (RM-DIC)

Keisuke Haseda, Keita Kanematsu, Yoshihiro Ohta (Div. Biotechnology and Life sciences, Tokyo University of Agriculture and Technology)

Mitochondria produce ATP by utilizing the membrane potentials. In this study, we measured the mitochondrial internal density with RM-DIC and examined the influence of the internal density on mitochondrial membrane potentials (MMP). Both the internal densities and the MMPs are heterogeneous in a single cell. Though it is considered that increase in the internal densities affects the MMPs through the concentration of proton pumps, the correlation between them remains to be clarified. To examine the effects of the mitochondrial internal densities on the MMPs, we have improved RM-DIC and examined each mitochondrion in terms of the optical length and TMRE fluorescence as measures of the internal density and MMP, respectively. To eliminate the effects of the mitochondrial size on the optical length and TMRE fluorescence intensity, we corrected these values with the mitochondrial sizes obtained from transmittance images of individual mitochondria. When we measured isolated mitochondria, the corrected optical length showed a close correlation with the corrected TMRE fluorescence. These results suggest that the increase in internal densities of mitochondria stimulates mitochondrial activity. Next we examined mitochondria in neurites but we did not observe the correlation between the internal density and the activity. These results indicate that the surrounding environments of mitochondria are more important for the mitochondrial activity than the internal density.

\section{PS042 ${ }^{19} \mathrm{~F}$ MRI monitoring of Gene Expression in living cells}

Hisashi Matsushita ${ }^{1}$, Shin Mizukami ${ }^{1,2}$, Yuki Mori ${ }^{2,3}$, Fuminori Sugihara ${ }^{1,2}$ Masahiro Shirakawa ${ }^{4}$, Yoshichika Yoshioka ${ }^{2,3}$, Kazuya Kikuchi ${ }^{1,2}\left({ }^{1}\right.$ Grad. Sch Eng., Univ. Osaka, ${ }^{2}$ Immunology Frontier Research Center, Univ. Osaka, ${ }^{3}$ Grad. Sch. Frontier Biosci., Univ. Osaka, ${ }^{4}$ Grad. Sch. Eng., Univ. Kyoto)

It is highly significant to accurately determine spatiotemporal expression patterns of each gene in vivo for the establishment of new medical treatments, development of diagnostic methods, and discovery of pharmaceuticals. The development of ${ }^{19} \mathrm{~F}$ MRI probes as a powerful in vivo imaging tool for monitoring biological phenomena has attracted increasing attention, since ${ }^{19} \mathrm{~F}$ MRI has many advantages such as no detectable intrinsic MRI signals in biological samples and the relatively high sensitivity, which corresponds to $83 \%$ of ' $\mathrm{H}$ sensitivity.

Recently, we successfully developed off/on switching ${ }^{19} \mathrm{~F}$ MRI probes to detect enzyme activities on the basis of the paramagnetic relaxation enhancement (PRE) effect. In the previous study, we detected $\beta$-galactosidase activity as reporter enzyme for gene expression in the cytosol using a specific ${ }^{19} \mathrm{~F}$ MRI probe. However, the cells required fixation to image intracellularly expressed $\beta$ gal activity, because the probe molecules were not permeable through the intact plasma membrane. For the in vivo applications, it is necessary to detect gene expression in living cells. In this report, we describe a novel method for monitoring gene expression in living cells by measuring extracellularly expressed enzyme activity by ${ }^{19} \mathrm{~F}$ MRI. We constructed a new imaging system by exploiting $\beta$-lactamase displayed on the plasma membrane as a reporter enzyme, a ${ }^{19} \mathrm{~F}$ MRI probe was rationally developed to monitor $\beta$-lac activity.

\section{PS043 Development of nanocapsule probes for sensitive ${ }^{19} \mathrm{~F}$ MRI}

Hiroaki Mukai ${ }^{1}$, Shin Mizukami ${ }^{1,2}$, Yosuke Nakanishi ${ }^{1}$, Fuminori Sugihara ${ }^{2}$, Kazuya Kikuchi ${ }^{1,2}$ ( ${ }^{1}$ Grad. Sch. Engin., Osaka univUniv., ${ }^{2}$ iFReC)

MRI is suitable for in vivo imaging because it can non-invasively visualize the living body with high spatial and temporal resolution. Thus, recently many smart ${ }^{1} \mathrm{H}$ MRI probes have been reported. However, such ${ }^{1} \mathrm{H}$ MRI signal enhancement needs to be discriminated from the background ${ }^{1} \mathrm{H}$ MRI signals of water, fatty acids, and other biomolecules. To avoid the limitation, we have focused on the use of ${ }^{19} \mathrm{~F}$ MRI. Since almost no intrinsic ${ }^{19} \mathrm{~F}$ MRI signals are observed in animal bodies, a high signal-to-noise ratio measurement of extrinsic ${ }^{19}$ F-containing compounds could be achieved. Thus, we have developed the ${ }^{19} \mathrm{~F}$ MRI probes to detect protease activity on the basis of PRE effect. " However, further improvement of the sensitivity is still required for practical usage. Thus, we developed nanoparticles (NPs) containing perfluorocarbon as highly sensitive ${ }^{19} \mathrm{~F}$ MRI contrast agents.

When the nanoparticles were injected into live animals, the ${ }^{19} \mathrm{~F}$ NMR signals were clearly detectable and accumulation was observed in the liver after 1 day. Protein-tag ligands were modified on the surface of NPs, then specific cellular labeling was achieved. In summary, we developed a highly sensitive ${ }^{19} \mathrm{~F}$ MRI nanoparticle that can be visualized in vivo, and this novel material would lead to novel molecular imaging method using ${ }^{19} \mathrm{~F}$ MRI.

1) J. Am. Chem. Soc., 2008, 130, 794-795

3PS044

\section{hiPSCs 細胞培養のための polydimethylsiloxane への細胞外基 質コート}

ECM coating on polydimethylsiloxane for hiPSCs culture

Ryosuke Yoshimitsu', Koji Hattori ${ }^{2}$, Shinji Sugiura ${ }^{2}$, Toshiyuki Kanamori ${ }^{2}$, Kiyoshi Ohonuma ${ }^{1}$ ( Nagaoka University of Technology, ${ }^{2}$ National Institute of Advanced Industrial Science and Technology) 
Human induced pluripotent stem cells (hiPSCs), which can be cultured in monolayers under a feeder- and serum-free condition, provide a good model for studying cell differentiation by imaging. However, cell differentiation on a conventional culture dish faces problems such as spatio-temporal unevenness of medium components caused by autocrine and paracrine factors and by intermittent medium change. To address such problems, we have developed a perfusion culture microchamber array chip that enables the control of culture conditions. Most previously reported microchips have been made of polydimethylsiloxane (PDMS). However, only limited cell types are cultivable on PDMS without extracellular matrix (ECM) coating. Here, we show that ECM coating (fibronectin, laminin, collagen, and gelatin) is determined by the adhesion, growth, and differentiation of hiPSCs on the PDMS surface under a feeder- and serum-free condition. The number of adhered cells and the growth rate of hiPSCs that were cultured on the PDMS surface coated with fibronectin and laminin were larger than that on the surface coated with collagen and gelatin. Flow cytometry analysis revealed that undifferentiated marker positive cells on the PDMS surface coated with fibronectin and laminin were larger than on the one coated with collagen and gelatin. These results suggest that the optimal ECM for culturing hiPSCs on a PDMS surface is fibronectin and laminin. This study enables the establishment of differentiation process imaging systems using hiPSCs on perfusion culture chips.

\section{PS045 Perfusion device to observe of single ES cells}

Shougo Nakamura ${ }^{1}$, Atushi Maruyama ${ }^{4}$, Yuichi Wakamoto ${ }^{2}$, Shin-ichi Sakai $^{3}$, Bayar Hexig ${ }^{3}$, Toshihiro Akaike ${ }^{3}$, Kiyoshhi Ohnuma ${ }^{4}$ ( ${ }^{1}$ Nagaoka university of Technology, ${ }^{2}$ Grad. Sch. Arts. \& Sci., Univ. Tokyo, ${ }^{3}$ Grad. Sch. Biosci. \& Biotech., Tokyo Inst of Tech, ${ }^{4}$ TRI, Nagaoka Univ of Tech)

ES and iPS cells characterized by both pluripotency and self-renewal are a promising source of regenerative medicine, as well as a good model for studying cell transition to multiple states. It is believed that cell population differentiation is a result of individual cell differentiated sub-population. To determine which hypothesis is more reasonable, we developed a single cell observation system, which we reported at the previous meeting. The system consists of three elements: GFP reporter mouse ES cells used to monitor the differentiation state, serum- and feeder-free culture conditions, and an E-Cad-Fc coated plate that enables us to dissociate cells into single cells. However, the number of data acquired is very small, because cells at seeding were heterogeneous in their state, and many cells were lost during the medium change. In this study, we developed a perfusion device for observing a single-cell reaction to medium stimulation to improve data acquisition efficiency. This device has two functions: to change the culture medium automatically by using a perfusionculture system, and to seed the cells directly in the device using a cell sorter. This device may enable us to elucidate mechanisms of cell transtion to multiple states.

\section{PS046 アルギン酸シートを用いた初代培亚細盷セルソーティング法の開発}

A Non-destructive Culturing and Cell Sorting Method for Cardiomyocytes and Neurons Using an Alginate Layer

Hideyuki Terazono' ${ }^{1}$, Hyonchol Kim ${ }^{1}$, Akihiro Hattori ${ }^{2}$, Fumimasa Nomura ${ }^{2}$, Tomoyuki Kaneko", Kenji Yasuda ${ }^{1,2}$ ( ${ }^{1}$ Yasuda "On-chip cellomics" project, KAST, ${ }^{2}$ Biomed. Info., Biosys., Biomat. Bioeng., Tokyo Medical and Dental Univ.)

It is proposed that a non-destructive collecting method of cultured cells after identifying their functional characteristics. Embryonic stem (ES) or induced Pluripotent stem (iPS) cells are widely expected to be used in clinical therapeutics for transplantation or as drug screening tools. For each passaging and collecting of cells, cultured cells are usually detached from the culture dish using collagenase or trypsin, which degrades the extracellular matrix or proteins. These methods are harmful to cells, so that fragile cells cannot be recultured. Alginate is a useful polymer for use as a culturing scaffold, because it is non-perturbing to cells. In our method, cells are cultivated on a thin alginate layer in a culture dish and released by spot application of a calcium chelate buffer that locally melts the alginate layer and allows collection of cultured cells at the single-cell level. Primary hippocampal neurons, beating human embryonic stem (hES) cell-derived cardiomyocytes, and beating hES cellderived cardiomyocyte clusters cultivated on an alginate layer were successfully released and collected with a micropipette. The collected cells were recultured while maintaining their physiological function, including beating, and elongated neurites. The results suggest that this method may allow to sort cells that physiological properties are aligned, and eventually facilitate transplantation of matured ES- or iPS-derived cardiomyocytes and neurons differentiated in culture.

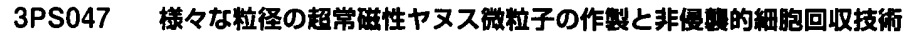
への応用

Fabrication of Superparamagnetic Janus Particles Having Various Sizes and Its Application for Non-Destructive Cell Sorting

Hyonchol Kim ${ }^{1}$, Hideyuki Terazono ${ }^{1}$, Hiroyuki Takei ${ }^{1,2}$, Kenji Yasuda ${ }^{1,3}$ $\left({ }^{1}\right.$ KAST, ${ }^{2}$ Facul. Life Sci., Toyo Univ., ${ }^{3}$ Inst. Biomat. Bioeng., Tokyo Med. Dent. Univ.)

Janus particles, which are composed of two chemically distinct hemispheres, are used for sensors, new devices, or for a model to study self-assembly physics. Fabrication of various Janus particles is therefore fundamental, and we propose a method for fabrication of superparamagnetic Janus particles and its application for target cell sorting without serious damages. Janus particles were fabricated using polystyrene spheres as casts. The sphere was placed on a substrate, and magnetic materials were deposited on the sphere by thermal evaporation. In addition, thin Au layer, typically 2 to $5 \mathrm{~nm}$ thick, was formed on the particle for easy immobilization of biomolecules. Janus particles whose half was composed of deposited metal and the other was polystyrene were then fabricated. By using this method, we fabricated superparamagnetic Janus particles with strict control of magnetic material thickness. One advantage of our method is easy fabrication of particles having various sizes using different polystyrene casts, which achieve particle size-dependent collection of target molecules. For a practical application of fabricated magnetic particles, a DNA aptamer was immobilized onto the particle to use it for specific cell sorting. The particle was reacted with cells and collected using magnet, then its target cell can be specifically collected. Moreover, the magnetic probe can be easily detached from the cell with nuclease treatment. These results indicate that fabricated magnetic particles can be used for non-destructive cell sorting.

\section{PS048一細胞時系列解析を用いた定昷的プロモータ活性評価法の開叱}

Event driven time lapse image analysis reveal the promoter activity

Kazumi Hakamada, Yusaku Somei, Jun Miyake (Osaka University)

Modeling and understanding cellular dynamics is the holy grail of biology. Many researchers addressed to this target and developed a lot of methods. It is still difficult to elucidate cellular dynamics quantitatively due to cellular heterogeneity. Here, using time lapse images of both phase-contrast and fluorescent images of single cell image analysis, we investigate the promoter activity. HeLa cells were transfected with AcGFP, which encodes AcGFP under the control of promoter of CMV, EF1- $\alpha$, and SV40 by NanoJuice, respectively. After transfection of them, phase-contrast image and fluorescent images of cells in each well were recorded at interval of ten min for $24 \mathrm{~h}$ by using Programmable Cellular image Tracer. By recording the timing of cell division by using phase contrast image and that of onset of fluorescent by using fluorescent image, we calculate the difference between cell division and onset of gene expression about three promoters. The fluorescent intensity was observed in order of CMV (248.5 min), EF1- $\alpha$ (461.3 $\mathrm{min})$, SV40 (339.4 $\mathrm{min})$, respectively and time periods between cell division and onset of three promoters had statistically significance ( $p<1.8 \times 10-20$, welch's t-test). Because these three vectors have the same genes (AcGFP) and they were expected to have the same translation period, those differences suggested the differences of promoter activities. By using single cell time lapse image analysis, we can quantitatively compare the promoter activity.

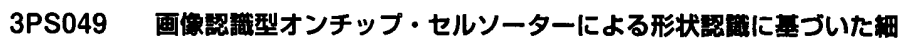 胞分醀法の定且的評価}

Quantitative evaluation of cell separation method based on shape recognition using on-chip imaging cell sorter

Akihiro Hattori ${ }^{1,2}$, Tomoyuki Kaneko ${ }^{1}$, Fumimasa Nomura ${ }^{1}$, Kenji Yasuda ${ }^{1,2}$ ( ${ }^{1}$ Dept. Biomedical Information, Div. Biosystems, Inst. Biomaterials and Bioengineering, Tokyo Medical ana Dental Univ., ${ }^{2}$ Yasuda "On-chip Cellomics "Project, Kanagawa Academy of Science and Technology)

We have evaluated the method for the label-free optical microscopic image-based separation of cardiomyocytes from the mixture of collagenase-digested embryonic mouse heart cells using the on-chip imaging cell sorter system, in which real-time high-speed camera-based phase-contrast cell image recognition was performed. To separate the cardiomyocytes from other cells including 